\title{
Large Vocabularies for Keypoint-Based Representation and Matching of Image Patches
}

\author{
Andrzej Śluzek \\ Khalifa University, Abu Dhabi, UAE \\ andrzej.sluzek@kustar.ac.ae
}

\begin{abstract}
In large visual databases, detection of prospectively similar contents requires simple and robust methods. Keypoint correspondences are a popular approach which, nevertheless, cannot detect (using typical descriptions) similarities in a wider image context, e.g. detection of similar fragments. For such capabilities, the analysis of configuration constraints is needed. We propose keypoint descriptions which (by using sets of words from large vocabularies) represent semi-local characteristics of images. Thus, similar image patches (including similarly looking objects) can be preliminarily retrieved by straightforward keypoint matching. A limited-scale experimental verification is provided. The approach can be prospectively used as a simple mid-level feature matching in large and unpredictable visual databases.
\end{abstract}

Keywords: keypoint description, keypoint correspondences, visual vocabulary, near-duplicate patches, affine invariance.

\section{Introduction}

In large visual databases of unknown and unpredictable contents, one of the critical problems is a fast detection of prospectively similar pieces of data. BoW techniques are relatively successful in retrieval of near-duplicate images (using descriptions of detected keypoints) and the-same-category scenes (using dense sampling instead of keypoint detection).

Retrieval of near-duplicate patches (located on different backgrounds) is another important problem in visual datasets. The results can be used for detection of similar objects located in random scenes, for the identification of typical/popular objects in visual collections, etc. Unfortunately, it is still a challenging problem, primarily because of its computational complexity. In random images, keypoint correspondences seldom indicate locations which are similar in a wider context. Thus, typical methods combine keypoint matching with the analysis of configuration constraints (often modeled by affine transformations). Such methods have limited applicability to large databases because each pair of images has to be separately processed to identity groups of similar keypoints satisfying the configuration constraints.

Configuration constraints have been analyzed using diversified techniques. RANSAC-based algorithmss ([12] $)$, hashing ([34]), the Hough transform $([\underline{56}])$, 
topological constraints $([7,8])$ are examples of the most popular approaches. Nevertheless, if the verification of constraints is needed, the size of processed visual databases cannot grow beyond certain limits (in spite of recent improvements, e.g., 910]).

Our goal, motivated by the recent results presented in [11, is to introduce and evaluate an alternative representation of individual keypoints. We propose large vocabularies of words invariantly characterizing keypoints in a semi-local context (i.e. a word represents both a keypoint and its neighborhood). By using such vocabularies, similar patches can be prospectively identified by straightforward keypoint matching. At the low level, we use typical tools, i.e. Harris-Affine keypoint detector ([12]) and SIFT descriptor (513]), but any affine-invariant keypoint detector and any keypoint descriptor can be alternatively applied.

Section 2 of the paper contains fundamentals of the proposed approach. First, we briefly review TERM features, their descriptors and the corresponding vocabularies introduced in [1] to affine-invariantly characterize configurations of elliptical keypoints. TERM3 features (the main tool used in [11]) are built from triplets of keypoints. This is a disadvantage because even in images with artificially reduced numbers of keypoints, the numbers of TERM3 can be very large. Therefore, we show in Subsection 2.2 how to embed similar data into descriptions of individual keypoints using several novel vocabularies.

A limited-scale experimental validation is presented in Section 3. First, it is verified how reliably a match between two keypoints indicates that these keypoints belong to near-duplicate patches. Secondly, we investigate whether images with near-duplicate patches can be retrieved using just a presence of keypoint correspondences as the criterion. The results on publicly available databases are not perfect, but they are encouraging. Section 4 concludes the paper.

\section{Principles of the Method}

\subsection{TERM Features and Their Descriptors}

TERM3/TERM2 features, [11, characterize configurations of three/two elliptical keypoints. Given $E_{0}$ ellipse (with $p_{0}$ origin) and two external points $p_{1}$ and $p_{2}$ (which are origins of two other ellipses $E_{1}$ and $E_{2}$ ) we can build a trapezoid using the intersections of $E_{0}$ with $\left[p_{0}, p_{1}\right]$ and $\left[p_{0}, p_{2}\right]$ vectors, and with the diameters parallel to the corresponding tangent lines (Fig. 11).

Similar trapezoids are built in $E_{1}$ and $E_{2}$ ellipses. Then, such triplets of $Q_{0(12)}$, $Q_{1(02)}$ and $Q_{2(01)}$ trapezoids are refereed to as TERM3 features for three-ellipse configurations (Figs 2 a,b,c,d). In order to avoid ambiguities, the ellipses are arranged in a tuple according to their areas.

If the ellipses are jointly transformed by an affine mapping, the configuration of trapezoids is correspondingly reshaped, Figs $2 \mathrm{~b}, \mathrm{~d}$. Thus, similar values of affine-invariant descriptors of TERM3 would indicate the affine covariance of three-ellipse configurations. 


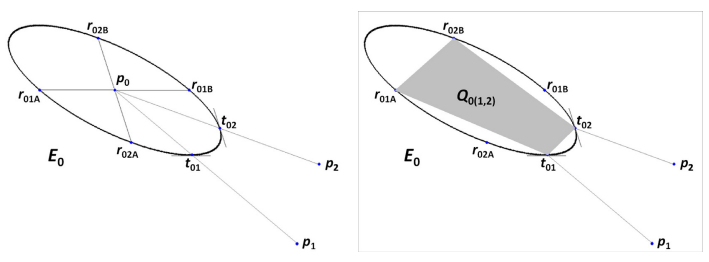

Fig. 1. A trapezoid built in $E_{0}$ ellipse based on two external points $p_{1}$ and $p_{2}$

Affine-invariant TERM2 features (which play only a supplementary role) consist of three quadrilaterals $Q_{T}, Q_{B}$ and $Q_{L}$ (see the bottom row of Fig. 2) defined by a configuration of two ellipses.

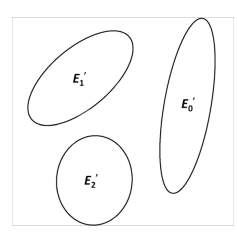

(a)

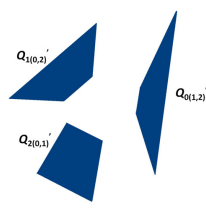

(b)

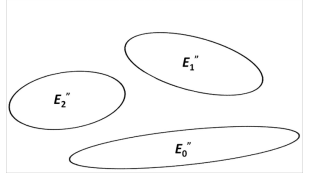

(c)

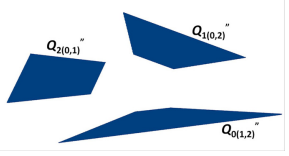

(d)
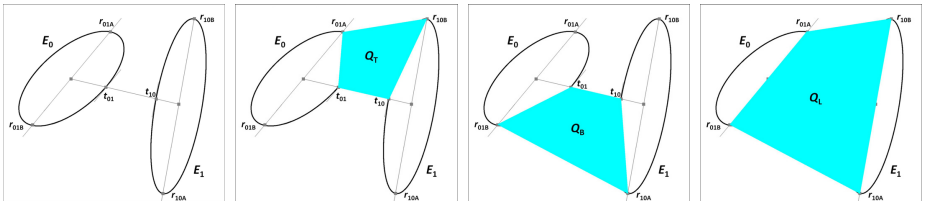

Fig. 2. Principles of TERM3 features(a,b,c,d) and TERM2 features (bottom row)

Descriptors of TERM3 and TERM2 features (referred to as T3 and T2, correspondingly) are built using a simple affine-invariant moment expression Inv $=\frac{\mu_{20} \mu_{02}-\mu_{11}^{2}}{\mu_{00}^{4}}$, where $\mu_{p q}$ indicates a central moment of $(p+q)^{t h}$ order. T2 descriptor consist of the Inv values computed separately for $Q_{T}, Q_{B}$ and $Q_{L}$ quadrilaterals (i.e. T2 is a $3 D$ vector). For T3, the values of $I n v$ are obtained for individual trapezoids $Q_{0(12)}, Q_{1(02)}$ and $Q_{2(01)}$, for their pairs and for the union of all of them. Therefore, T3 is a $7 D$ vector.

High performances of TERM3 matching in retrieval of near-duplicate patches are reported in 11. The most successful variant (over $90 \%$ of correct matches) is a combination of T3, T2 and SIFT words, i.e. two TERM3 are matched if: (a) their T3 words are identical, (b) SIFT words of the contributing keypoints are correspondingly identical, and (c) T2 words for pairs of contributing keypoints are also correspondingly identical. Fig. 3 shows an example of correspondences found by such a method (the standard O2O SIFT-based matching is also presented for a reference). 


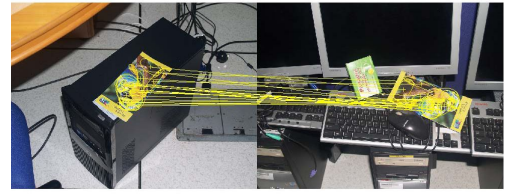

(a)

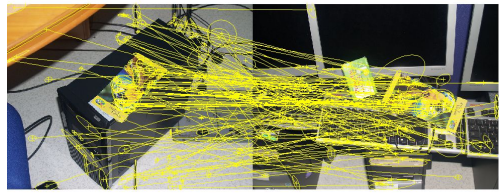

(b)

Fig. 3. Exemplary correspondences found by TERM3 matching (a) and keypoint matching by using SIFT-based mutual nearest neighbor O2O (b)

\subsection{Vocabularies for Keypoint Description and Matching}

The major disadvantage of TERM3 is the number of such features in typical images. Even if the numbers are reduced (first, by reducing the numbers of keypoints and, secondly, by building the features over keypoint neighborhoods of limited size) more than 10,000 TERM3 can be frequently formed in images containing just 200-300 keypoints. As an alternative, we propose the incorporation of TERM-related data into descriptions of individual keypoints. Then, although the complexity of keypoint description increases, the number of records remains the same and all extracted keypoints can be used (if necessary).

Consider a TERM3 feature with the largest (dominant) $E_{0}$ elliptical keypoint. As explained earlier, its description includes:

- T3 word of the whole TERM3 feature;

- SIFT words of the dominant ellipse $E_{0}$ and the other ellipses $E_{1}, E_{2}$;

- T2 words of TERM2 features built over $\left(E_{0}, E_{1}\right),\left(E_{0}, E_{2}\right)$ and $\left(E_{1}, E_{2}\right)$ pairs of ellipses.

Assume the cardinalities of SIFT, T3 and T2 vocabularies are, correspondingly, $\|S V\|,\left\|T V_{3}\right\|$ and $\left\|T V_{2}\right\|$. Then, $E_{0}$ keypoint can be described by various vocabularies (only selected options are mentioned) representing both the keypoint and the configuration of its neighborhood:

- VocA: SIFT word of $E_{0}$. Two keypoints are matched if they have the same SIFT words. $\|V o c A\|=\|S V\|$. Each keypoint is described by a single word only (in this case no neighborhood data are used).

- VocB: SIFT word of $E_{0}$ and SIFT word of either $E_{1}$ or $E_{2}$. Two keypoints are matched if they have the same SIFT words and some of their neighbors have the same SIFT words. $\|V o c B\|=\|S V\|^{2}$. Each keypoint is described by a set of words (SoW). A similar idea was proposed in the pioneer work 7 .

- VocC: SIFT word of $E_{0}$ and T3 word TERM3. Two keypoints are matched if they have the same SIFT words and they belong to TERM3 features with the same T3 words. $\|V o c C\|=\|S V\| \times\left\|T V_{3}\right\|$. Each keypoint is described by SoW.

- VocD: SIFT word of $E_{0}$, SIFT word of either $E_{1}$ or $E_{2}$ and T3 word of TERM3. Two keypoints are matched if they have the same SIFT words, some of their neighbors have the same SIFT words and these neighbors belong to 
TERM3 features with the same T3 word. $\|V o c D\|=\|S V\|^{2} \times\left\|T V_{3}\right\|$. Each keypoint is described by SoW.

- VocE: SIFT words of $E_{0}, E_{1}$ and $E_{2}$, T3 word of TERM3, T2 words of TERM2 built over $\left(E_{0}, E_{1}\right),\left(E_{0}, E_{2}\right)$ and $\left(E_{1}, E_{2}\right)$. Two keypoints are matched only if they are dominant keypoints of two TERM3 matched according to Subsection 2.1. $\|V o c E\|=\|S V\|^{3} \times\left\|T V_{3}\right\| \times\left\|T V_{2}\right\|^{3}$. Each keypoint is described by SoW.

In general, two keypoints are considered a match (within a selected vocabulary) if their SoW's ( sets of words) intersect. The objective of the conducted experiments is to evaluate applicability of keypoint matching defined by such vocabularies to near-duplicate patch detection and to verify how effective this simple approach is in the retrieval of images containing near-duplicate patches.

\section{$3 \quad$ Experiments}

The main experiment has been conducted using a publicly available VISIBLE 1 dataset which consists of 100 images containing diversified views on varying backgrounds of 1, 2 or 3 objects from over 30 types of objects. Because the objects are approximately piecewise planar, outlines of their shapes can be naturally used as the ground truth for near-duplicate patches (see examples in Fig. 44). With 100 images, there are 4,950 image pairs in this dataset, and 512 pairs share at least one near-duplicate patch. The objective is to detect/locate such patches and, subsequently, to retrieve pairs of images containing near-duplicate patches.
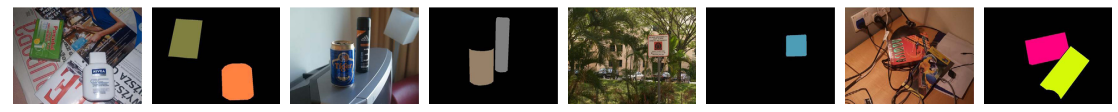

Fig. 4. Exemplary images and the outlines of near-duplicate patches from VISIBLE dataset

In the other experiment, images from popular databases (PASCAL2007, Caltech101, 15 Scenes, INRIA, etc.) have been used. Unfortunately, in these images the required ground truth is not available (e.g., in PASCAL2007 outlines of thesame-class objects are provided, but classes are defined semantically so that such outlines seldom share near-duplicate patches). Thus, the results of this experiment are used for a general evaluation rather than for a systematic analysis of performances.

We are certainly aware of a limited scale of the conducted experiments. However, databases where the ground truth is provided as outlines of near-duplicate patches (instead of the-same-class objects) are not easily available (we hope to build such a database but this is an extremely tedious process).

${ }^{1}$ http://www.ii.pwr.wroc.pl/ visible/data/upload/FragmentMatchingDB.zip 


\subsection{Methodology}

Keypoints are extracted using Harris-Affine detector, [12]. The SIFT vocabulary for keypoint description contains 500 words (i.e. $\|S V\|=500$ ) built from almost 1,000, 000 keypoints found in 800 diversified images. In the preprocessing phase, TERM features are formed in all images. We basically follow the methodology of [1] where more details can be found. Similarly to many other works (e.g. 6 14 15]), neighborhoods of limited size are considered (with a non-standard definition of neighborhoods which include only keypoints of sizes proportional to the distance between keypoints). The small-size vocabularies describing TERM features are built using over 30,000,000 triplets of keypoints from 1000 images. T3 vocabulary contains 128 words $\left(\left\|T V_{3}\right\|=128\right)$ and there are only 27 words in T2 vocabulary $\left(\left\|T V_{3}\right\|=27\right)$. Thus, the cardinalities of the proposed vocabularies are: $\|V o c A\|=500,\|V o c B\|=250,000,\|V o c C\|=64,000$, $\|V o c D\|=32,000,000$ and $\|V o c E\|=314,928,000,000,000$. Note that some vocabularies require long integer representation.

With large numbers of image pairs to be matched, it is almost impossible to manually verify the ground truth of keypoint correspondences. Thus, we use an approximate method where a match is considered a true correspondence if in both images the keypoints belong to the outlines of near-duplicate patches. This may generate some false positives (matching different parts of near-duplicates) and false negatives (similar fragments which are outside the outlined patches) but, in general, the results have been found fairly accurate.

Therefore, performances of keypoint matching can be measured using only precision. The results of image pair retrieval are evaluated using three popular metrics, i.e. precision, recall and F-measure. Instead of the ordinary F-measure, we use $F_{\beta}$ (with a small value $\beta=0.3$ ) which highlights a higher importance of precision. It can be argued that in very large databases users are more interested in fairly small sets of mostly relevant objects (even if some relevant objects are not retrieved) than in large sets of mostly irrelevant objects (even though almost all relevant objects are included).

\subsection{VISIBLE Database}

TERM3 matching has been tested on VISIBLE database in 11. Using limited numbers of keypoints to reduce the numbers of TERM3 features (which, nevertheless, exceed 10,000 in some images) a high $93 \%$ precision of feature matching is obtained. We first match the same keypoints represented by the sets of words proposed in Subsection 2.2. Note that the numbers of data records are now very small (all images contain less than 300 keypoints). The results for selected vocabularies are given in Table 1 (in one case we use the intersection of correspondences provided by two vocabularies).

It can be noted that matching by VocE (which incorporates all data used to match TERM3) is almost as good as TERM3 matching; a minor deterioration is because we limit the size of SoW's to 100 (while actually some keypoints appear in more than 100 TERM3). However, even for smaller vocabularies the results 
Table 1. Precision of feature matching for selected vocabularies ([11] results provided for reference)

\begin{tabular}{|l|c|c|c|c|c|c|c|}
\hline Vocabulary & VocA & VocB & VocC & VocB $\cap$ VocC & VocD & VocE & 11 \\
\hline total matches & $2,202,689$ & 434,774 & 148,667 & 90,970 & 53,862 & 616 & n.a. \\
\hline correct matches & 75,429 & 28,528 & 9,532 & 8,902 & 6,643 & 555 & n.a. \\
\hline precision & $3.64 \%$ & $6.56 \%$ & $6.41 \%$ & $9.78 \%$ & $12.33 \%$ & $90.10 \%$ & $93 \%$ \\
\hline
\end{tabular}

can be improved if we accept only matches with sufficiently large intersections of SoW's. For example, using 5 as the threshold, precision of VocB, VocB $\cap$ VocC and VocD increases to $44 \%, 70 \%$ and $41 \%$, correspondingly.

Subsequently, we retrieve a pair of images as prospectively containing nearduplicate patches if at least one keypoint match is found. For VocB, VocC, VocB $\cap$ VocC and VocD, a keypoint match requires at least 5 elements in SoW intersections. For VocE any non-empty intersections are accepted. Again, we use the 11] results as the reference compared to the results provided in Table 2] It should be noted that VISIBLE dataset contains 512 image pairs sharing similar objects/patches but only 311 of them share any keypoints within the outlines of patches (because of a limited number of keypoints used). Thus, recall of image pair retrieval cannot exceed $311 / 512=60.74 \%$ and the recall values in Table 2 should be seen in this context.

Table 2. Retrieval of image pairs sharing near-duplicate patches

\begin{tabular}{|l|c|c|c|c|c|c|c|}
\hline Vocabulary & VocA & VocB & VocC & VocB $\cap$ VocC & VocD & VocE & 11 \\
\hline returned & 4950 & 340 & 3685 & 31 & 747 & 259 & 272 \\
\hline correct & 311 & 92 & 210 & 162 & 135 & 233 & 248 \\
\hline precision & $6.68 \%$ & $27.06 \%$ & $5.70 \%$ & $48.94 \%$ & $18.07 \%$ & $89.96 \%$ & $91.17 \%$ \\
\hline recall & $60.74 \%$ & $17.97 \%$ & $41.02 \%$ & $31.64 \%$ & $26.37 \%$ & $45.51 \%$ & $48.23 \%$ \\
\hline$F_{0.3}$ & $7.21 \%$ & $25.98 \%$ & $6.13 \%$ & $46.83 \%$ & $18.55 \%$ & $83.25 \%$ & $84.98 \%$ \\
\hline
\end{tabular}

When we considered ALL extracted keypoints (note that for the 11] method it would be prohibitively costly) the results are qualitatively similar (see Tables 3 for selected details). The precision values might be slightly lower (with the corresponding increase of recall) because less prominent keypoints contribute a number of additional correspondences (often incorrect).

Table 3. Selected results by using ALL keypoints extracted in VISIBLE images

\begin{tabular}{|l|c|c|c|c|}
\hline Vocabulary & keypoints matched(correct) & precision & image pairs (correct) & precision $/$ recall $/ F_{0.3}$ \\
\hline VocA & $9,027,281(190,145)$ & $2.11 \%$ & $4950(510)$ & $10.30 \% / 99.61 \% / 11.12 \%$ \\
\hline VocB $\cap$ VocC & $40,941(22,748)$ & $55.56 \%$ & $653(263)$ & $40.27 \% / 51.37 \% / 41.01 \%$ \\
\hline VocD & $25,459(10,847)$ & $42.61 \%$ & $795(138)$ & $17.36 \% / 26.95 \% / 17.88 \%$ \\
\hline
\end{tabular}


Altogether, we can conclude that vocabularies incorporating TERM-related data into words describing individual keypoints are prospectively a useful tool for a preliminary search in large and complex databases. VocE vocabulary is clearly superior (because it inherits many properties of the original TERM3 matching). However, if the memory footprint is an issue, VocB $\cap$ VocC can be alternatively used (with the corresponding drop of performances). Exemplary matches (including one example which is partially incorrect) obtained by using VocE are given in Fig. 5.
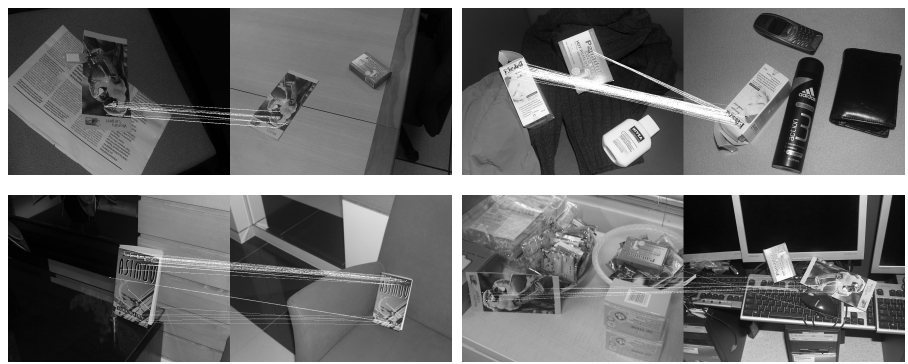

Fig. 5. Exemplary matches in VISIBLE dataset by using Method D

\subsection{Other Databases}

A random collection of over 1,000 images from several popular databases have been used in the second experiment. Initially, we hoped that near-duplicate patches would be retrieved from pairs of images sharing the-same-class objects, especially for man-made objects with some configurational consistency (e.g. cars, trains, etc.) but is has not been confirmed. In PASCAL2007 (where the ground truth outlines of the-same-class objects are available) precision of image pair retrieval was at the level of random choice. Nevertheless, some conclusions can be drawn from almost 500,000 image matches performed (although only some of them have been later visually inspected).

The general observations support results of the first experiment. In particular, approx. 90\% of keypoint correspondences (returned by both VocE and VocB $\cap$ VocC matching) actually represent visually identifiable similar patches. If clusters of several correspondences are found, images usually contain larger patches. A few examples (shown either locally or in the image context) are provided in Fig. 6. 


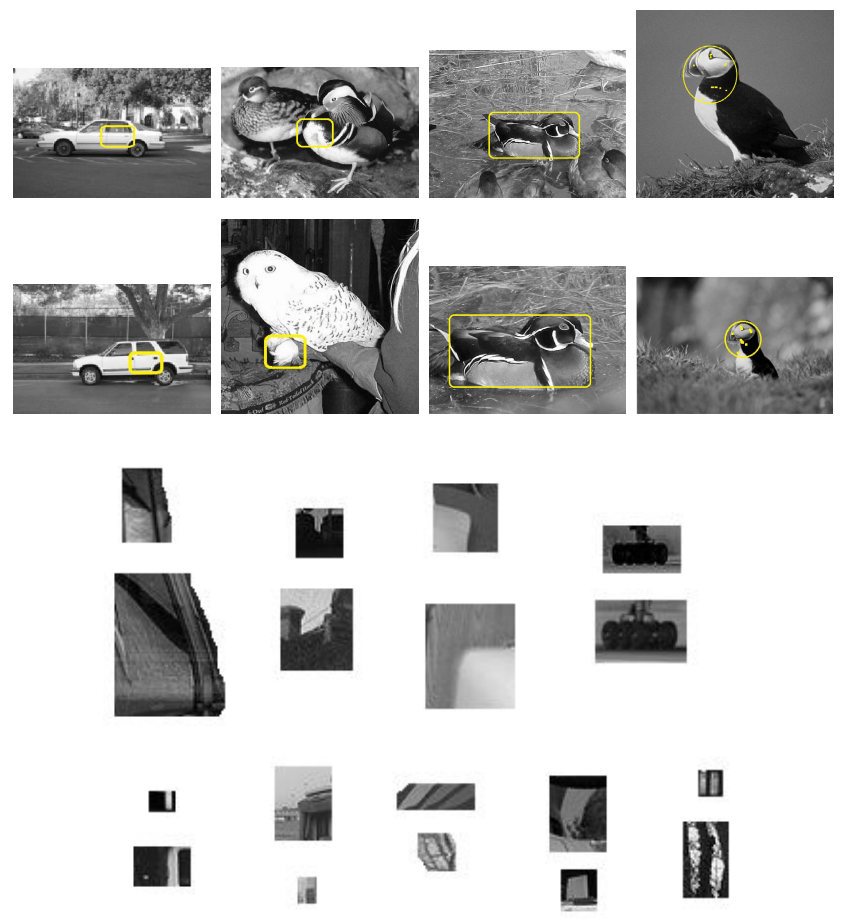

Fig. 6. Exemplary retrievals of near-duplicate patches shown in the context of whole images (top rows) and locally (bottom). The outlines are approximate.

\section{Final Remarks}

The paper argues that detection of near-duplicate patches in images of random contents is feasible at the level of individual keypoint matching (i.e. without any verification/analysis of configuration constraints). Large-size vocabularies are proposed for this purpose (each keypoint is described by a set of words). In contrast to other keypoint-related large vocabularies (e.g., 4]) we use words representing both visual and geometric characteristics of keypoint neighborhoods,

The preliminary experimental verification has been conducted on a small database (which provides the ground truth in the required form). A larger-scale evaluation (without the ground truth data) on image from popular databases provides qualitatively similar results. Because of its very low computational costs (word-based matching), the method can be instrumental in building mid-level features (e.g. [16]) for large databases and in visual data mining, [17]. 


\section{References}

1. Fischler, M., Bolles, R.: Random Sample Consensus: a Paradigm for Model Fitting with Applications to Image Analysis and Automated Cartography. In: Buxton, B.F., Cipolla, R. (eds.) ECCV 1996. LNCS, vol. 1064, pp. 683-695. Springer, Heidelberg (1996)

2. Chum, O., Matas, J.: Matching with prosac - progressive sample consensus. In: Proc. IEEE Conf. CVPR 2005, San Diego, CA, pp. 220-226 (2005)

3. Wolfson, H., Rigoutsos, I.: Geometric hashing: An overview. IEEE Comp. Science and Engineering 4, 10-21 (1997)

4. Chum, O., Perdoch, M., Matas, J.: Geometric min-hashing: Finding a (thick) needle in a haystack. In: Proc. IEEE Conf. CVPR 2009, pp. 17-24 (2009)

5. Lowe, D.G.: Object recognition from local scale-invariant features. In: Proc. 7th IEEE Int. Conf. Computer Vision, vol. 2, pp. 1150-1157 (1999)

6. Paradowski, M., Śluzek, A.: Local Keypoints and Global Affine Geometry: Triangles and Ellipses for Image Fragment Matching. In: Kwaśnicka, H., Jain, L.C. (eds.) Innovations in Intelligent Image Analysis. SCI, vol. 339, pp. 195-224. Springer, Heidelberg (2011)

7. Schmid, C., Mohr, R.: Object recognition using local characterization and semilocal constraints. Technical report, INRIA (1996)

8. Tell, D., Carlsson, S.: Combining Appearance and Topology for Wide Baseline Matching. In: Heyden, A., Sparr, G., Nielsen, M., Johansen, P. (eds.) ECCV 2002, Part I. LNCS, vol. 2350, pp. 68-81. Springer, Heidelberg (2002)

9. Perd'och, M., Chum, O., Matas, J.: Efficient representation of local geometry for large scale object retrieval. In: Proc. IEEE Conf. CVPR 2009, pp. 9-16 (2009)

10. Jegou, H., Douze, M., Schmid, C.: Improving bag-of-features for large scale image search. International Journal of Computer Vision 87, 316-336 (2010)

11. Śluzek, A., Paradowski, M.: Detection of Near-Duplicate Patches in Random Images Using Keypoint-Based Features. In: Blanc-Talon, J., Philips, W., Popescu, D., Scheunders, P., Zemcik, P. (eds.) ACIVS 2011. LNCS, vol. 7517, pp. 301-312. Springer, Heidelberg (2012)

12. Mikolajczyk, K., Schmid, C.: Scale and affine invariant interest point detectors. International Journal of Computer Vision 60, 63-86 (2004)

13. Lowe, D.G.: Distinctive image features from scale-invariant keypoints. International Journal of Computer Vision 60, 91-110 (2004)

14. Schmid, C., Mohr, R.: Local grayvalue invariants for image retrieval. IEEE Trans. PAMI 19, 530-535 (1997)

15. Yang, D., Śluzek, A.: A low-dimensional local descriptor incorporating tps warping for image matching. Image and Vision Computing 28, 1184-1195 (2010)

16. Boureau, Y.L., Bach, F., LeCun, Y., Ponce, J.: Learning mid-level features for recognition. In: Proc. IEEE Conf. CVPR 2010, pp. 2559-2566 (2010)

17. Han, J.: Data mining for image/video processing: A promising research frontier. In: Proc. Int. Conf. on Content-based Image and Video Retrieval CIVR 2008, pp. 1-2 (2008) 